\title{
Abstracts of presentations at the congress of the Critical Care Society of Southern Africa, July 2015
}

\section{ORAL PRESENTATIONS}

\section{Intensivists' practices and perceptions of HIV testing in South African intensive care units \\ D Singh}

University of the Witwatersrand, University of KwaZulu-Natal

singhd6@ukzn.ac.za

Background. Testing for HIV infection requires informed consent. Little guidance is given in the case of incapacitated patients who cannot consent but who may benefit from earlier diagnosis and directed therapy. Also, little is known regarding intensivists' perceptions and practices concerning ethical and legal aspects of HIV testing.

Objective. To ascertain intensivists' practices and ethical perceptions of diagnostic HIV testing in South African intensive care units (ICUs).

Method. A semi-structured online questionnaire was emailed to 47 intensivists at university hospital ICUs probing HIV testing practices and policies, ethical and legal perceptions, and views regarding available legal and ethical guidelines, surrogate consent, and the disclosure of the test results.

Results. The response rate was $51 \%$. The majority of ICUs did not have a policy or protocol in place. The majority of respondents considered unconsented HIV testing ethical and beneficial in certain critically ill patients, as early diagnosis may help guide management. The majority also felt that current testing guidelines were inadequate, that surrogate consent for testing was not reliable, and that test results not be disclosed except in specific circumstances.

Conclusion. The majority of South African ICUs lack a policy for HIV testing. However, physicians perceive HIV testing as beneficial in specific circumstances. Guidelines regarding testing based on patient autonomy are perceived as not in the patient's best interests. Surrogate consent for testing is viewed as unreliable and it may be preferable to perform unconsented testing at the discretion of the intensivist.

\section{A review of transplantation activity in South Africa (1991 - 2011)}

DA Thomson, ${ }^{*}$ E Muller, F McCurdie, D Kahn

University of Cape Town, Groote Schuur Hospital

*david.thomson@uct.ac.za

Background. South African organ transplantation has evolved with limited government oversight across two health systems.

Objective. To analyse trends in organ transplant practices across the hospitals, provinces and healthcare systems in South Africa from 1991 to 2011.

Method. Statistics submitted to the Organ Donor Foundation were analysed as to organs transplanted, living or deceased donation, paediatric or adult recipients, location and whether publicly or privately funded.
Results. A total of 7280 transplants were performed across 10 public and 13 private hospitals in 5 provinces with the majority (84\%) being kidney transplants. Deceased donors decreased from 4.2 per million population (pmp) in 1991 to $1.7 \mathrm{pmp}$ in 2011. An increase in living related donation kept transplant numbers relatively constant. Initially, the majority of kidney (91\%) and all heart and liver transplants were in the public sector. By 2011, the majority of kidney (56\%), heart (96\%) and liver (80\%) transplants were in the private sector. Organ trafficking from 1999 to 2003 increased numbers temporarily. KwaZulu-Natal, where this practice came to light, showed a decrease in transplants from 110 in 2003 to 5 in 2010. Paediatric transplants comprised $2.6 \%$ of all transplants.

Conclusion. Deceased donation has decreased markedly in South Africa. There has been a marked shift towards the private sector and increased government support is required to ensure equitable access to organ transplantation.

\section{The effect of body position on regional distribution of ventilation and muscle activity in infants and children}

A Lupton-Smith, ${ }^{1 *}$ A Argent, ${ }^{1}$ B Morrow, ${ }^{1}$ P Riemensberger ${ }^{2}$

1 University of Cape Town

${ }^{2}$ Geneva University Hospital

*aluptonsmith@gmail.com

Background. Recent studies have questioned the pattern of ventilation distribution (VD) in the spontaneously breathing paediatric population. There are no recent studies examining the effect of body position in mechanically ventilated infants and children. There are also few studies reporting respiratory muscle activity in relation to body position in this population.

Objective. To determine the effect of body positions on regional VD and diaphragmatic muscle activity in mechanically ventilated children.

Method. Thoracic electrical impedance tomography (EIT) measurements and surface electromyography (sEMG) measurements of hemidiaphragm activity $(\mathrm{V})$ were taken in left and right side lying positions in mechanically ventilated infants and children. Functional EIT images were produced offline and total regional relative tidal impedance in the left and right lungs was calculated for each patient in each position.

Results. Preliminary data on the first 17 patients (aged 6 months to 6 years) are presented. Eleven (65\%) children demonstrated varied patterns of VD between left and right side lying. No significant differences were found between left and right lungs in left $(p=0.99)$ and right $(p=0.56)$ side lying. There was no significant difference in hemidiaphragmatic activity between positions.

Conclusion. The paediatric pattern of ventilation during mechanical ventilation is not predictable. Diaphragm activity may not be affected by side lying positions. 


\section{Parent perception of quality of care in a South African paediatric intensive care unit (PICU)}

\author{
B Morrow $_{1}{ }^{1 *} \mathrm{C} \mathrm{Mol,}{ }^{2} \mathrm{M}$ van Dijk, ${ }^{2}$ A Argent ${ }^{3}$ \\ 'Department of Paediatrics and Child Health, University of Cape Town \\ ${ }^{2}$ Departments of Paediatric Surgery and Paediatrics, Erasmus \\ MC-Sophia Children's Hospital, The Netherlands \\ ${ }^{3}$ Department of Paediatrics, University of Cape Town and Red Cross War \\ Memorial Children's Hospital \\ *brenda.morrow@uct.ac.za
}

Background. The effect of physical, psychosocial and environmental factors on perceived quality of PICU care has been poorly studied in South Africa.

Objective. To explore how parents of children admitted to PICU perceive the current environment and quality of care.

Method. Parents/guardians of stable children admitted to PICU completed the locally adapted EMPATHIC-30 questionnaire developed by Latour et al. (2013). This questionnaire assesses parental satisfaction with provided care in the PICU context. Institutional Research Ethics Committee approval was obtained.

Results. 100 questionnaires were completed, $77 \%$ by mothers. Thirty-five percent of children's admissions were unplanned and $88 \%$ were mechanically ventilated. Teamwork: $95 \%$ reported that doctors and nurses worked well and efficiently together. Information: $91 \%$ responded that information provided about their child's condition and management was clear. Care: $86 \%$ and $94 \%$ felt nurses and doctors were concerned about their child's comfort; $91 \%$ felt staff were respectful. Doctors' and nurses' performance was rated as 9.6 (1.5) and 8.9 (1.4) (mean (standard deviation)) on a 10-point scale. Environment: $97 \%$ reported the PICU was clean; $85 \%$ felt there was sufficient space around the bedside; $78 \%$ reported acceptable noise levels.

Conclusion. The experience of PICU care by parents of critically ill South African children was generally positive.

\section{Screening critically ill patients with an adapted early mobility readiness protocol ensures safety of a therapeutic early mobility position}

E Conradie $_{,}{ }^{1 *}$ C Fourie, $^{2}$ S Hanekom ${ }^{1}$

'Department of Physiotherapy, Stellenbosch University

${ }^{2}$ Surgical Unit, Tygerberg Hospital

*vanheerden.elmarie@gmail.com

Background. The effect of the 45 semi-recumbent position on the haemodynamic stability of critically ill patients is questionable. This routine nursing position could minimise the negative effects bedrest has on the cardiac and pulmonary systems.

Objective. To evaluate the feasibility of an adapted early mobility readiness protocol and the effect of a therapeutic early mobility position on two haemodynamic parameters: the mean arterial pressure (MAP) and central venous oxygen saturation $\left(\mathrm{ScvO}_{2} \%\right)$.

Methods. Twice weekly, all patients nursed in the surgical and respiratory units were screened with the protocol. Patients who passed the protocol and inclusion criteria were tested in the baseline nursing position followed by the testing position. Haemodynamic parameters were measured at 0,3 and 10 minutes.

Results. We screened 138 patients. Eleven patients passed, male/ female (9/2) with a median age of $47(20-67)$ years. The mean MAP
(95\% confidence interval (CI)) increased from 95.87 (91.72 - 100.03) $\mathrm{mmHg}$ to $97.86\left(94.16\right.$ - 101.57) $\mathrm{mmHg}$ and the mean $\mathrm{ScvO}_{2} \%(95 \% \mathrm{Cl})$ values increased from $81.43(79.43-83.42) \%$ to $82.06(79.43-84.20) \%$ when the baseline nursing position was followed by the testing position. Conclusion. Patients who passed the protocol were not physiologically challenged when placed in the testing position. The protocol can be used by clinicians to identify patients suitable for the testing position. Work is needed to investigate the outcome of patients nursed in this position.

\section{Plasma glutamine levels in adult intensive care unit patients}

A Nienaber ${ }^{1 *}$ RC Dolman, ${ }^{1}$ AE Van Graan, ${ }^{1}$ R Blaauw ${ }^{2}$

${ }^{1}$ Centre of Excellence for Nutrition, North-West University, Potchefstroom Campus

${ }^{2}$ Division of Human Nutrition, Stellenbosch University

*aristahefer@yahoo.com

Background. Glutamine (GIn) deficiency is an independent predictor of mortality in intensive care unit (ICU) patients and its supplementation is recommended for proven outcome benefits. Recent data suggest that early GIn supplementation increases mortality in certain patient groups.

Objective. To investigate plasma GIn levels of adult ICU patients and to determine relationships between Gln levels, gender, diagnosis and selected inflammatory markers.

Methods. This cross-sectional study included 60 mixed adult ICU patients, paired with 60 healthy controls (CG). Gln levels of patients were compared to that of $C G$ and the relationship between $\mathrm{Gln}$ levels and Interleukin-6 (IL-6) or C-reactive protein (CRP) was examined. A non-parametric ROC curve was computed to determine the CRP concentration cut-off above which Gln becomes deficient.

Results. ICU patients had significantly lower Gln levels than CG $(496 \mathrm{~mol} / \mathrm{L} \mathrm{v.} 718 \mathrm{~mol} / \mathrm{L}, p<0.0001)$. Of the patients, $38.3 \%(n=23)$ had deficient $(<420 \mathrm{~mol} / \mathrm{L})$ and $6.7 \%(n=4)$ had supra-normal Gln levels ( $>930 \mathrm{~mol} / \mathrm{L})$. Gln was inversely associated with CRP $(r=-0.44$, $p<0.05)$ and IL-6 $(r=-0.23, p=0.08)$ levels. A CRP cut-off value of $95.5 \mathrm{mg} / \mathrm{L}$ was determined above which $\mathrm{Gln}$ levels became deficient. Conclusion. ICU patients had lower Gln levels compared with healthy controls; not all patients were Gln deficient and some presented with supra-normal levels. This highlights the importance of selecting patients for Gln supplementation. Since GIn levels were inversely associated with CRP, the latter might be useful as a proxy marker for Gln status.

\section{Hypoxaemia on arrival in a multidisciplinary intensive care unit}

$K$ de Vasconcellos, ${ }^{*} D$ Singh, DL Skinner

King Edward VIII Hospital and University of KwaZulu-Natal

*kimdevasconcellos@gmail.com

Background. Transport of the critically ill patient poses the risk of numerous complications. Hypoxaemia is one such serious adverse event and is associated with potential morbidity and mortality. It is however potentially preventable.

Objective. To determine the incidence of hypoxaemia on arrival in a tertiary multidisciplinary intensive care unit (ICU) and to identify risk factors for this complication.

Method. Retrospective observational study using data collected during a prospective transport audit at King Edward VIII Hospital from May 2013 to February 2014. 
Results. Hypoxaemia occurred in 23/148 (15.5\%) admissions. Statistically significant risk factors for hypoxaemia on univariate analysis $(p<0.05)$ included lack of $\mathrm{SpO}_{2}$ monitoring, transfer by an intern as opposed to other medical/paramedical staff, and transfer from internal medicine. Use of neuromuscular blockers and transfer from theatre were protective. Binary logistic regression analysis revealed lack of $\mathrm{SpO}_{2}$ monitoring to be the only significant independent predictor of hypoxaemia (OR=5.59 (95\% confidence interval 1.33 - 23.57), $p=0.019$ ).

Conclusion. Hypoxaemia is common on admission to ICU and could potentially be prevented by simple interventions such as appropriate transport monitoring.

\section{The physiotherapy management of thoracotomy patients: A survey of current practice in Gauteng}

\section{Schwellnus $L_{1}^{*}$ Roos R, Naidoo V}

University of the Witwatersrand

*grassman.liezel@gmail.com

Background. Physiotherapy is an essential clinical component in the management of patients after thoracic surgery to prevent respiratory complications and improve mobility. To date, published literature is scarce regarding physiotherapy practice in this area.

Objective. To establish the physiotherapy modalities used in the management of thoracotomy patients during all phases of recovery. Method. A cross-sectional study of 1389 physiotherapists registered with the South African Society of Physiotherapy in Gauteng was conducted. A self-administered questionnaire was distributed electronically. The data collection period was 2 months and data were analysed with descriptive statistics.

Results. Three hundred and twenty-three physiotherapists (23.3\%) responded. Preoperative physiotherapy management was determined by the patients' risk profiles and consisted of information and respiratory techniques. Prophylactic postoperative management was high. The modalities used most commonly were respiratory techniques, e.g. deep breathing exercises $(97.6 \% ; n=83)$ and exercise interventions, e.g. early mobilisation $(95.3 \% ; n=81)$, trunk $(85.9 ; n=73)$ and upper limb mobility exercises $(91.8 \% ; n=78)$. Pain-reducing modality use was less common, e.g. transcutaneous electrical nerve stimulation $(12.9 \% ; n=11)$. Post-hospital physiotherapy was uncommon (32.6\%; $n=46)$.

Conclusion. Physiotherapists in Gauteng use techniques to prevent and manage postoperative pulmonary complications in patients who undergo thoracic surgery. Education is needed regarding the importance of effective pain management by physiotherapists for these patients both during and after hospital stay.

\section{POSTER PRESENTATIONS}

\section{Use of proton pump inhibitors in the ICUs of three academic hospitals in Johannesburg}

N Biyase, ${ }^{*} \mathrm{H}$ Perrie, J Scribante, S Chetty

Department of Anaesthesia, University of the Witwatersrand

*nana.biyase@yahoo.com

Background. Stress ulcer profylaxia (SUP) is an important part of management of critically ill patients in intensive care units (ICUs). However, inappropriate use of these drugs has important clinical implications such as ventilator-associated pneumonia and gastrointestinal tract infections. The overuse of proton pump inhibitors (PPIs) SUP is a rapidly growing problem internationally.

Objectives. To describe the use of SUP in ICUs and to compare the appropriate use of PPIs versus the inappropriate use according to the risk factors of the patient as per American Society of Hospital Pharmacists guidelines.

Method. A retrospective, descriptive, contextual study design was used. A 3-month audit of ICU charts of adult patients admitted to ICUs at Chris Hani Baragwanath Academic Hospital, Charlotte Maxeke Johannesburg Academic Hospital and Helen Joseph Hospital that fulfilled the inclusion criteria.

Results. A total of 174 patients were included in the study. Of these patients, 156 were started on SUP, $60.9 \%$ (95) of them were appropriately started on SUP and $39.1 \%$ (61) were inappropriately on SUP. There was overuse of SUP of $39.1 \%(61)$. The number of patients who qualified for SUP came to 113 . In that group only $28.3 \%$ (32) were on PPIs and the remainder of the patients $71.7 \%$ (81) were either on other agents or were not started on SUP, reflecting an underuse of PPIs of $71.7 \%$ (81).

Conclusion. Our study found inappropriate overuse of SUP but underuse of PPIs where they should have been used.

\section{Outcome and severity of surgical patients admitted to a non-tertiary multidisciplinary critical care unit}

O Swart, ${ }^{*}$ R Duvenage

Worcester Provincial Hospital

*oostewalt.swart@westerncape.gov.za

Background. Demand for critical care services exceeds availability. Limited published public health sector critical care unit (CCU) data, outside of academic institutions, are available. Worcester Provincial Hospital's (WPH) 5-bed CCU is classified as a high-care unit ( $\mathrm{HCU})$ and services a rural population of approximately 600000 in the Cape Winelands East and Overberg district of the Western Cape.

Objective. To describe the severity and in-hospital all-cause mortality of surgical patients admitted to the WPH CCU.

Method. A descriptive study of surgical patients admitted to WPH CCU collected prospectively from January to December 2014.

Results. The WPH CCU had 114 (8 re-admissions) surgical admissions out of a total of 610 during 2014. The mean (standard deviation (SD)) age and median length of stay were 46.0 (SD 18.7) years and 2 (range 0.5 - 13.5) days, respectively. The mortality rate was $17.0 \%$ (18/106) with a mean Apache II score of 11.1 (SD 6.6). Ventilatory support was required in $68.4 \%$ (78/114) of admissions with 156 ventilation and 67 continuous positive airway pressure days. Ventilation and inotropic support (total of 70 inotrope days) was required in $28.9 \%(33 / 114)$ of admissions, indicating multiorgan failure. Only $18.9 \%(20 / 106)$ of patients were referred to tertiary CCUs.

Conclusion. Disease severity and mortality were similar to the only non-tertiary, although non-surgical, CCU data published. Almost a third of the CCU admissions had multi-organ failure that is deemed to be beyond the scope of an HCU. 
A comparison of excess fluid to be removed in haemodialysis patients, as estimated by haemodialysis staff versus multiple frequency bioelectrical impedance analysis

J Downs, ${ }^{*}$ F Veldman, S Kassier

Dietetics Department, King Edward VIII Hospital and Discipline of Dietetics \& Human Nutrition, School of Agriculture, Earth \&

Environmental Sciences, University of KwaZulu-Natal

*jane.downs3@gmail.com

Background. Currently, in most haemodialysis (HD) units in South Africa, the excess fluid to be removed in HD is estimated by the staff who compare the previous post-HD versus the subsequent pre-HD body weight (wt). Bioelectrical impedance analysis (BIA) has been shown to have clinical value and may potentially protect the HD patients from risks associated with under- and over-hydration.

Objective. To compare excess fluid to be removed in HD as estimated by the staff versus the volume measured per multiple frequency BIA.

Methods. A prospective, non-randomised observational study was conducted. Repeated measures of 24 BIA pre- and post-HD measurements were taken over 3 months on 20 chronic HD subjects (50\% male; ages 21 - 63 years) at the King Edward VIII Hospital HD unit. Results. There was a significant difference between the excess fluid measured per BIA v. the staff estimate. Subjects with a body fat $(\mathrm{BF})>35 \%$ and a $\mathrm{BMI}>28 \mathrm{~kg} / \mathrm{m}^{2}$, had a negative $3 \mathrm{rd}$ water space v. those with lower BF and BMls.

Conclusion. Excess fluid measured per BIA v. staff estimates differed. Previous studies have shown HD patients with a higher BF and BMI have a lower mortality risk; we postulate that the negative 3rd water space in overweight subjects is due to greater fluid and sodium lost in perspiration.

\section{Outcome of children admitted to a combined paediatric/neonatal ICU in a low to middle income country}

D Ballot, ${ }^{*}$ V Davies, P Cooper

University of the Witwatersrand

*daynia.ballot@wits.ac.za

Background. In South Africa, limited resources result in a lack of sufficient ICU staff and facilities for patient demand. Doctors use the ethical principle of distributive justice to ration scarce intensive care unit (ICU) facilities. The mortality rate in paediatric ICU (PICU) is variable and reports range between $5 \%$ and $14.6 \%$. Only $19.6 \%$ of all ICU beds in both the private and public sectors in South Africa were dedicated to paediatric and neonatal intensive care. Charlotte Maxeke Johannesburg Academic hospital (CMJAH) has a combined paediatric and neonatal ICU with 14 ventilator beds.

Objective. To establish the short-term outcome of children admitted to CMJAH paediatric and neonatal ICU between 1 January 2013 and 31 December 2014.

Methods. This study is a retrospective descriptive review of children admitted to the CMJAH PICU. Information was obtained from the CMJAH PICU and neonatal databases at CMJAH.

Results. A total of 1073 patients were admitted to PICU at CMJAH during the study period: 472 paediatric patients, 245 very low birth weight (VLBW) neonates and 356 other neonates. Over one-third of the patients were surgical $(414 / 1073 ; 38.5 \%)$. The overall mortality rate was 24.3\% (261/1 073): 100 (40.8\%) for VLBW, 91 (25.5\%) for other neonates and $70(14.8 \%)$ for paediatric patients

Conclusions. There is a high demand for PICU beds. Separate paediatric and neonatal ICUs are justified. Mortality of PICU patients is within the reported range.

\section{Predicting mortality rates: Hospital Standardised Mortality Ratio v. APACHE IV R Toua, ${ }^{*} \mathrm{~J}$ De Kock \\ University of Cape Town and Mediclinic \\ *rene.toua@mediclinic.co.za}

Background. Mortality is widely used as a qualitative measure. However, actual mortality rates can't be directly compared and to accurately and simply predict the risk of death is a big challenge.

Objective. To correlate predicted mortality as calculated with an administrative model (Hospital Standardised Mortality Ratio) to a physiological model (APACHE IV): combined cohort and samples stratified by physiological prediction level $(<10 \%$ predicted mortality, $10-50 \%$ predicted mortality or $>50 \%$ predicted mortality).

Method. Cross-sectional: 40 private healthcare group hospitals (68 critical care units). One-way analysis was done. A total of 47982 critical and high-dependency patients were scored from 1 June 2013 to 31 July 2014. 1921 records (0.4\%) were excluded due to missing values, duplicate records and values not within parameters $(n=46061)$.

Results. Correlation was moderate for the combined cohort (Pearson's correlation index 0.62 (95\% confidence interval (CI) 0.62 -0.63), R-squared 0.38); verygood for the $<10 \%$ stratum (Pearson's correlation index 0.88 , R-squared 0.78 (95\% Cl $0.878-0.882)$ ); good for the $10-50 \%$ predicted mortality rates (Pearson's correlation index 0.78 , R-squared $0.61(95 \% \mathrm{Cl} 0.77-0.79)$ ) and no correlation for the $>50 \%$ predicted mortality stratum (Pearson's correlation index 0.09 , R-squared 0.01 (95\% Cl $0.03-0.15)$ ).

Conclusion. The administrative predictive model is not suitable for predicting mortality in the highest stratum.

\section{Profile of ICU bed requests at Helen Joseph Hospital}

H Hurri, ${ }^{*}$ S Chetty, J Scribante, H Perrie

University of the Witwatersrand

*hurrih@gmail.com

Background. Intensive care unit (ICU) beds are a scarce resource at Helen Joseph Hospital (HJH). A limited number of beds serve a population with a large burden of disease. Medical practitioners request ICU beds for patients they deem in need of ICU; however, the decision to admit into ICU remains with the ICU consultant on call, consultants being from different disciplines. No formal triage such as APACHE or SAPS scoring or admission guidelines is currently in place. Objective. To compile a profile of the ICU admission requests at $\mathrm{HJH}$. Method. A contextual, prospective, descriptive research design was followed in this study. Data were collected during one winter and one summer month in 2012.

Results. A total of 139 patients were included. The median age was 44 years. The majority of patients (79\%) were under the age of 60 years. The overall admission rate was $35.25 \%$ and the most common reason for admission was mechanical ventilation. Reasons for refusal were $41 \%$ assessed as too ill, $30 \%$ assessed as too well and $29 \%$ were 
refused due to a lack of resources. Patients admitted to the ICU had a $77.55 \%$ survival rate. The relationship between ICU admission and 30-day outcome was statistically significant.

Conclusion. The lack of resources is problematic at HJH ICU. Survival rates correlate with international trends and triage methods appear to be effective.

\section{Are weight estimation methods applicable for rural African children? A comparative study}

\section{$\underline{\mathrm{ZNazo}}^{*}$}

Walter Sisulu University

*zandisile.nazo@gmail.com

Background. Accurate paediatric weight estimation is essential in the management of critically ill children as it is impractical to weigh these patients. The Advanced Paedaitric Life Support (APLS) formula has been used for weight estimation globally. Recent studies done in developed countries report that this formula significantly under-estimates children's weights, which may result in medical errors. Objective. To investigate the accuracy of APLS and Luscombe and Owen formulae in estimating weight of rural African children. To compare the performance of these formulae in this population.

Method. A prospective, observational and cross-sectional study involving a convenient population of children aged 1 - 10 years was conducted from September 2012 to March 2013. These children were attending a private paediatric practice and a public hospital paediatric outpatient department. They were weighed and weights recorded. Weight difference between the measured weight and the calculated weight for APLS and Luscombe and Owen formulae was the main outcome measure. The Bland-Altman method was used for detecting accuracy and precision of the formulae.

Results. A sample of 314 males and 252 females was analysed. There was no significant $(p>0.05)$ association between gender, actual weights and estimated weights. The mean values of actual weight of the private participants were significantly higher $(p<0.05)$ than those of public participants. Predictive accuracy was superior for the APLS than for the Luscombe formula. Using the mean percentage differences, the APLS again showed a superior advantage in predicting weight over the Luscombe formula. The estimated weight by the APLS formula within $10 \%$ error was higher than in the Luscombe formula. All results were statistically significant. Precision was more accurate with the APLS formula than with the Luscombe. Conclusion. The results showed that the APLS formula has a superior advantage over the Luscombe and Owen formula at estimating weight in this population of children. This is in agreement with the Western Cape study by Geduld et al.

\section{Patient perceptions of ICU care: A scoping review}

M van Ness, F Karachi, S Hanekom*

Physiotherapy Interdisciplinary Health Sciences, Stellenbosch University

*sdh@sun.ac.za

Background. Physiotherapy practice in intensive care unit (ICU)s is changing. Early mobilisation programmes are included and prioritised. Methods and measures to assess physiotherapy effectiveness in the ICU have often been geared to physiological data. It is unclear whether patients' perspective and satisfaction with care in ICU have been investigated.
Method. A scoping review was undertaken with the aim of determining how patient perception and satisfaction with critical care is measured. Seven databases were searched using the following keywords in variouscombinations: physiotherapy or physical therapy, patient satisfaction, perception or patient perception, patient experience, intensive care unit or ICU, critical care, hospitalised adult population, hospital, measurements, measuring and outcome measure.

Results. 1626 articles were independently screened by two reviewers at title, abstract and full text level respectively. The final review included 26 articles. Only two of the studies were conducted in Africa, compared with ten in Europe and six in Northern America, respectively. Nine of the included articles investigated a particular service such as nursing care, emergency care and physiotherapy with regards to patient perception and satisfaction. Only one article, published in 2008, investigated patient perception and satisfaction in physiotherapy. Various outcome measures were identified in this review that measure perception and/or satisfaction. However, there is currently no validated and reliable instrument to assess patient satisfaction with care in the ICU.

Conclusion. A gap in the literature was identified for patient perceptions regarding physiotherapy care in the ICU. The results will be used to inform the planning of a primary qualitative study. Knowing and understanding the patients' perception and satisfaction with care, ensures the professional development in the critical care field, and improving the quality of care.

\section{Articulating the nature of clinical specialist nurse practice}

JBell,* D van Rooyen, P Jordan

Stellenbosch University, Nelson Mandela Metropolitan University

*jbell@sun.ac.za

Background. People sharing stories of their encounters in critical care environments revealed that some nurses were perceived to have distinctive qualities that influenced an encounter in a positive way. Encounters with these nurses were described as being different and better, with the qualities that characterised anecdotes of 'different and better' nursing not experienced by all critical care nurses.

Objective. The purpose of this study was to articulate an understanding of the qualities that people who engage with critical care nurses recognise as 'different and better' to the norm of nursing practice they encounter in this discipline.

Method. Constructivist grounded theory methodology was used. Participants were drawn from patients' significant others, nurses and medical colleagues. Data generation began with participants contributing through in-depth unstructured individual interviews and creating a naïve sketch. A focused literature review contributed to a final study sample of 74 data items. Data generation was completed through grounded theory data analysis method processes. Results. An inductively derived substantive grounded theory named Being at Ease was constructed from the data. The core concern in recognising different and better nursing practice emerged as 'being at ease'. This is underpinned by four categories, : 'knowing self', 'skilled being', 'connecting with intention' and 'anchoring'.

Conclusion. Being at Ease adds to our practice narrative through this explanation of how tacit qualities of 'different and better' nursing are located as discrete elements within the complex nature of specialist clinical practice. 